\title{
Pressure management in smart gas networks for increasing hydrogen blending
}

\author{
Marco Cavana ${ }^{1, *}$, Enrico Vaccariello ${ }^{1}$, and Pierluigi Leone ${ }^{1}$ \\ ${ }^{1}$ Department of Energy, Politecnico di Torino, 10129 Torino, Italy
}

\begin{abstract}
The injection of hydrogen into existing gas grids is acknowledged as a promising option for decarbonizing gas systems and enhancing the integration among energy sectors. Nevertheless, it affects the hydraulics and the quality management of networks. When the network is fed by multiple infeed sites and hydrogen is fed from a single injection point, nonhomogeneous hydrogen distribution throughout the grid happens to lead to a reduction of the possible amount of hydrogen to be safely injected within the grid. To mitigate these impacts, novel operational schemes should therefore be implemented. In the present work, the modulation of the outlet pressures of gas infeed sites is proposed as an effective strategy to accommodate larger hydrogen volumes into gas grids, extending the area of the network reached by hydrogen while keeping compliance with quality and hydraulic restrictions. A distribution network operated at two cascading pressure tiers interfaced by pressure regulators constitutes the case study, which is simulated by a fluid-dynamic and multi-component model for gas networks. Results suggest that higher shares of hydrogen and other green gases can be introduced into existing distribution systems by implementing novel asset management schemes with negligible impact on grid operations.
\end{abstract}

\section{Introduction}

While there has been a consensus of fact that natural gas would have been the energy source for the transition, now that the decarbonization goals have become higher and aims to the complete decarbonization by 2050 , the natural gas industry is seen as a threat against a quicker transition towards the net-zero energy system.

However, the full electrification of the energy sector appears to be, if not unfeasible, not viable at all [1],[2]. There are sectors such as the transports (especially the heavy ones) and energy-intensive industries which are defined the "hard-to-abate" as the ones, for which molecule-based energy carriers appear to be the most viable solutions.

In this context, renewable gases such as green hydrogen and biomethane appear to be the perfect compromise between sustainability, storability, and flexibility of use. What is more, these gases are expected to be integrated within the current gas network system, contributing to enhancing the strategic value of the whole infrastructure, avoiding the risk of stranded assets.

Within renewable gases, hydrogen is the most promising one to become the energy vector of the future [3]. In the past few years, it has gained more and more momentum among stakeholders and institutions so that roadmaps at the national and European levels have been published [4]. In [4], the evolution of the gas network infrastructure towards a European-wide hydrogen backbone is described. The practice of blending hydrogen within the current natural gas flows is expected as a viable contribution to the market uptake of green hydrogen during the 2020 s.

However, hydrogen is to be considered an unconventional gas in the traditional natural gas system. A strong effort needs to be put in place for harmonization of natural gas regulation to include hydrogen and to allow cross-border exchanges. The latest achievement of the European Committee for Standardization (CEN) is the publication of the norm EN 16726:2019 [5] on standardization of gas quality (group H) which concludes the impossibility of setting a common limiting value, as presented in [6]. This shall go together with the research on the impact of hydrogen presence on the infrastructure's materials, valves and fittings as well as on the appliances of the final users.

Hydrogen blends up to $5-10 \%$ are commonly considered possible and already feasible. An extensive literature review across all the sectors of the natural gas system that might be impacted by hydrogen presence made by Marcogaz (European gas industry association) showed that criticalities are a few [7]. These appear among end-users such as compressed natural gas (CNG) vehicles and gas turbines. Problems with $\mathrm{CNG}$ vehicles are related to the onboard pressurized tanks which are made of high-strength steel for which a limit of $2 \% \mathrm{H}_{2}$ is set by the ISO Standard 11439. As for turbines instead, according to [8] which reviewed manufacturer's catalogs,

\footnotetext{
* Corresponding author: marco.cavana@polito.it
} 
the new Dry Low Emission turbines on the market may accept hydrogen up to $30 \%$. Still, problems with installed ones may exist.

For what concerns the household appliances, the ones installed after the 90s should fall within the Gas Appliances Directive (Directive 2009/142/EC) which foresees a test on flashback with a $77 \% / 23 \% \mathrm{CH}_{4}-\mathrm{H}_{2}$ mixture. About indoor piping, which is usually made of copper, hydrogen presence does not affect the integrity of the material [9]. In terms of safety, even though the flammability range of hydrogen and natural gas hydrogen blends is wider than the natural gas one, the Lower flammability limit of hydrogen blend is very similar to the one of natural gas. This factor, together with the fact that common odorants such as THT and TBM do not interact with hydrogen ([10] [11]) indicates that current procedures for natural gas may be suitable also for hydrogen blends.

As for the gas metering aspects, according to [12] gas meters do not experience significant metrological differences for concentration up to $20 \%$. In [13] a slight undercounting is registered but it is still within the range of the specification of EN 1359

Given the complexity and the diversity of stakeholders and appliances connected to the natural gas infrastructure, injection at the distribution level may be a useful starting point to demonstrate the feasibility of hydrogen blending while maintaining the impacts at a local level. Similar projects have already been set up in The Netherlands (2011) [14] and in UK (2019) [15]. Distribution system operators have to equip themselves with the expertise and tools to manage these innovative practices. Works on distributed injection of renewable gases have already been published. In [16], the distributed injection of hydrogen within a test case distribution network is performed. The pressure level is on the order of $75 \mathrm{mbar}$, thus representing a very lolcal portion of the network: it is representative of a neighbour. The case study aims at studying the impact of hydrogen injection on the pressure and gas flow fields with a steady state show case. In [17], a medium pressure (5 bar-g) infrastructure is addressed. The hydrogen injection scenario originates from a sector coupling tentative: a few hydrogen injection pattern and injection location have been tested in order to assess the hydrogen injection impact both in space and in time. Both works are based on the analysis of gas networks fed by only one citygates. Other works about gas network modelling focussing on distribution or regional networks may be found in [18] and [19]. Both assess multiple gas entry point networks, highlighting the potential to track gas quality of the network simulation softwares with multiple sources of renewable gases (i.e. biomethane and hydrogen).

Differently from the previous works, this one aims at combine the quality trascking fature and the fluiddynamic results of a in-house built network simulation software to highlight possible grid management measures in order to enhance the hydrogen injection and consumption within an existing infrastructure, while maintaining a maximum share of hydrogen in the blending.
In this work, the goal is to underline that the limited hydrogen receiving potential of a real distribution network can be increased by the correct management of pressure levels. Furthermore, by using a multi-component fluid-dynamic network model, the increasing complexity of gas quality distribution within the network is shown, highlighting the need for smart approaches to gas network management. With respect to the state-of-the-art approaches on distribution network management, the simulation tools here showcased allow the tracking of the hydrogen blending front throughout the whole network at steady-state conditions, as infeed pressure levels are varied. In the perspective of multi-gas networks, the tracking capability of different quality of gases will be fundamental for the correct allocation of energy consumption and final users' billing. What is more, the results show that by performing dynamic pressure regulation among the different regulation stations, it is possible to allow more or less renewable gas within the grid or to control which areas will be interested with blends and which others will not, giving a powerful tool to distribution system operators.

\section{Methodology}

The proposed technique of smart gas network management to increase the amount of hydrogen that is acceptably injectable within a gas network has been tested by means of a numerical simulation on an existing network asset.

\subsection{Gas network model}

The simulation of the gas networks consists of the calculation of the gas flow rates through all the pipelines of the infrastructure and the determination of the pressure level at all the ends of each pipeline (also called nodes of the networks) once all the outlet gas flow rates (at users' nodes) are defined and the pressures at the gas inlet nodes are given as boundary conditions. This is possible by jointly solving the equations of conservation of mass (the continuity equation) and conservation of momentum applied on each node and each pipeline of the network respectively. The closure of the mathematical problem is given by the equation of state which describes the thermodynamic behavior of the gas (or the mixture of gases) flowing within the infrastructure.

Given that the aim of this study is hydrogen blending and its propagation throughout the network, the multicomponent feature has been implemented so that the simulation tool is able to manage different gases within the same infrastructure. Natural gas is thus considered as a mixture of different chemical species whose concentration is defined at the inlet point of the network (given as further boundary condition) and calculated as a required output for all the nodes of the infrastructure.

The one dimensional (1-D) system of partial differential equations composed of the continuity and conservation of momentum equations has been simplified considering the following assumptions:

1) Isothermicity of the gas flowing in the pipelines; 
2) Horizontal pipes;

3) Negligible effect of the convective term (kinetic energy variation);

4) Steady-state condition.

These assumptions are commonly used in the literature [16],[20],[21], and by distribution system operators (DSOs) when running their commercial software for their business-as-usual activities. Thanks to these simplifications, the fluid-dynamic problem has been solved by the adaptation of the SIMPLE algorithm [22], which has been developed for incompressible fluids applications, to the case of networks of compressible fluids [23].

The specific case requires a network simulation tool that allows variable gas composition throughout the network caused by the presence of a hydrogen injection which is set to form a natural gas-hydrogen blend containing $10 \% \mathrm{~mol}$ of hydrogen. Thus, the boundary conditions of users' consumption are given in terms of thermal energy demand rather than flow rates of natural gas. This is due to the unknown distribution of the gas quality. At the hydrogen injection point, the inflow hydrogen flow rate is also given as a boundary condition, coming from the fixed condition of hydrogen share of the network blend. Starting from an initial guess in which the whole network is filled with $100 \%$ natural gas, the fluiddynamic solver is solved to define a first approximation of the pressure field and the gas flow rate throughout the network. Based on these tentative results, once the gas flow rates in all the branches are known, the solution of the "mixing problem" is possible. This consists of the solution of the conservation of mass for each chemical species of the gas flow for each node of the network, thus allowing the calculation of the updated gas composition at each node. This information allows the updating of the gas flow rates requested at each consumption node based on the updated heating value of the gas mixture, thus requiring an iteration of the fluid-dynamic problem. Using this new composition map as input thus, the fluiddynamic problem is solved iteratively until convergence is reached, meaning that a configuration of equilibrium regarding the gas composition map has been achieved and the steady-state of the network is fully represented.

\subsection{Case Study description}

An urban area of around 40,000 inhabitants, covering about $7.6 \mathrm{~km}^{2}$ has been considered as a case study. The area is served by a distribution network operated at two cascading pressure tiers interfaced by pressure regulators. The network is fed by three city booths (gas entry points) directly feeding the higher pressure tier of the infrastructure. The higher pressure tier is designed to be operated within a pressure range between 5 and 1.5 bar-g. It is composed of the main backbone to which two city booths out of three are connected and a shorter duct that connects the third entry points directly to a peripheral section of the lower pressure tiers distribution infrastructure.

The lower pressure tiers, which are designed to be operated within a pressure range between $0.5-0.04$ bar-g consists of two highly meshed infrastructures: the main one serving the main urban area and a much smaller one that serves a satellite urban conglomerate. The lower pressure network of the urban area is fed by six pressure regulators which are connected to the main higher pressure backbone plus one pressure regulator delivering the gas from the higher pressure duct connected to the third city booth. The lower pressure network of the satellite urban conglomerate is fed by a single pressure regulation station connected to the main higher pressure backbone by means of a branch pipe. The total number of pressure regulators in the distribution network is eight. In Figure 1 a distorted version of the network structure is given.

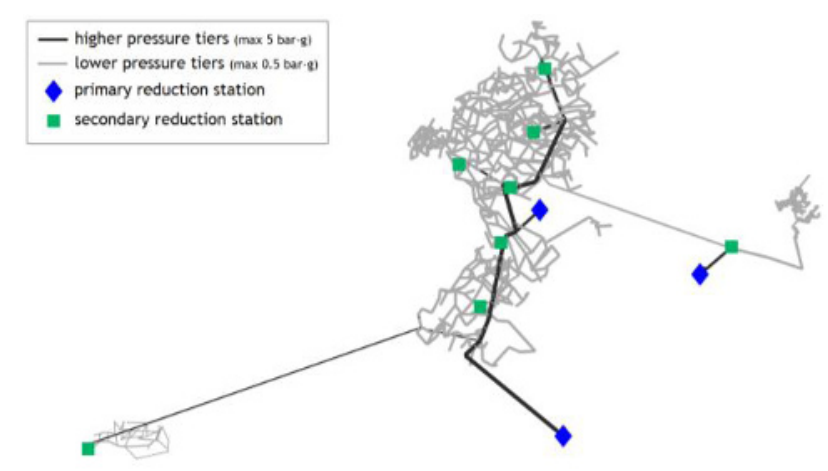

Fig. 1. Distorted representation of the topology of the distribution gas network infrastructure.

The total number of active gas meters in the area is around 10,300 and it has been assumed they are all connected to the lower pressure tiers of the network. It has been assumed that all the gas users are evenly distributed all over the lower pressure infrastructure: each node of the lower pressure distribution grid is associated with a number of gas meters and a consumption flow rate. It has also been assumed that all the gas users have the same gas consumption flow rate. All the simulated scenarios presented in this work share these homogeneity assumptions on the distribution of gas consumption.

The annual gas consumption of the whole area is 4.2 $\mathrm{MSm}^{3}$. The steady-state fluid-dynamic simulations have been performed referring to the expected peak gas consumption, which has been evaluated as about 3,900 $\mathrm{Sm}^{3} / \mathrm{h}$ for each gas user.

\subsection{Simulated scenarios}

The gas infrastructure as described in the previous paragraph has been modeled under the condition of hydrogen injection at a single point of the infrastructure. Furthermore, the hydrogen flow rate to be injected has been fixed by setting the maximum hydrogen share in the blend to $10 \%$ mol. Since the distribution network has three gas entry points, when keeping the same hydrogen injection position, the amount of hydrogen that is possible to inject depends on the fluid-dynamic 
configuration of equilibrium reached by the network depending on the pressure regulators settings.

At first, the gas network is simulated by setting the same value of the pressure set-point at the outlet of each reduction station. Then, the pressure set-point of the reduction station in which hydrogen blending is performed is increased. Furthermore, also the pressure set point of the secondary reduction station (feeding the lower pressure tiers) which is nearest to the hydrogen injection point is kept at a higher level than the others.

Variations of the distribution of the gas flow rates are observed, modifying the amount of hydrogen which can have access to the grid.

\section{Results}

The peak natural gas consumption of the whole area has been assumed to be equal to $3,900 \mathrm{Sm}^{3} / \mathrm{h}$, which

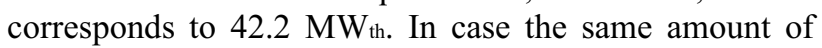
energy would be fed by a hydrogen-natural gas mixture with $10 \%$ hydrogen share, the total amount of hydrogen to be injected into the infrastructure would be $420 \mathrm{Sm}^{3} / \mathrm{h}$.

When dealing with a gas network having multiple gas entry points and choosing one single hydrogen injection point, the amount of hydrogen to be injected in order to form a gas mixture with a maximum hydrogen share equal to $10 \%$ is dramatically reduced.

The first network simulation has been conducted setting the pressure set-points of the three reduction stations which feed the higher pressure tier all equal to 3 barg. Similarly, the outlet pressure of all the secondary pressure reduction stations feeding the lower pressure tier has been set equal to 0.5 barg. The hydrogen injection is located at the reduction station labeled "C" in Figure 1. The fluid dynamic configuration of the network is such that the reduction station " $C$ " contributes to the fulfillment of the whole gas flow request by $12 \%$, being the minimum share with respect to reduction stations $\mathrm{A}$ and $\mathrm{B}$ which respectively contribute by $38 \%$ and $50 \%$. This implies that the amount of hydrogen that is possible to inject in the area is also reduced to $12 \%$ with respect to the value given above, being thus equal to $51.4 \mathrm{Sm}^{3} / \mathrm{h}$. As it is possible to observe in Figure 1, the spreading of the hydrogen-natural gas blend affects only a limited portion of the network: the tree-shaped branch to the right of the secondary reduction station following the reduction station "C" and part of the ducts flowing towards the meshed network receives exactly $10 \%$ hydrogen blend. This corresponds to $11 \%$ of the users of the network. A smaller portion of nodes and branches are instead reached by a mixture that underwent further blending, as an effect of the network topology. Thus, $3 \%$ of the users are reached by a hydrogen-natural gas mixture with $2 \%$ hydrogen share.

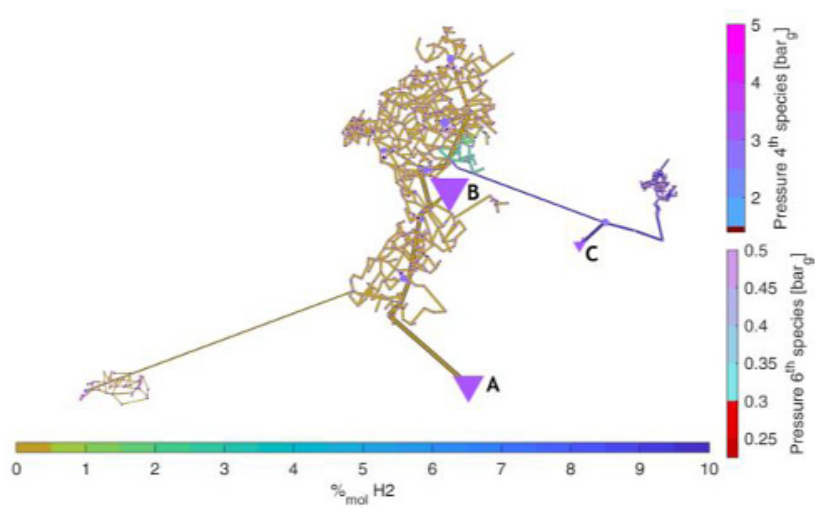

Fig. 2. Fluid-dynamic and quality tracking results of the first simulated scenario: all the pressure regulation stations have the same set-point.

By managing the pressure more smartly, it is possible to enhance the network's capability to receive and distribute hydrogen.

The second network simulation has been conducted by increasing the pressure set-point of the reduction station " $C$ " to the maximum admissible value: 5 barg. The other pressure reduction stations (A and $\mathrm{B}$ ) have been kept to $3 \mathrm{bar}$. As for the secondary reduction stations, the one directly connected to reduction station " $\mathrm{C}$ " has been kept a 0.5 barg set-point, while all the others have been set to 0.4 barg.

In Figure 2, the results of the fluid-dynamic simulation are given. While the pressure and velocity fields are still within the range of acceptability for the safe and correct operation of the network, the balance of gas flows has changed. In particular, the reduction station "C" contributes by $29 \%$ to the fulfillment of the total gas request in this case. The acceptable hydrogen flow rate is increased to $128.4 \mathrm{Sm}^{3} / \mathrm{h}$. As it is possible to see from Figure 2, the hydrogen is much more spread throughout the network. However, the highly meshed feature of the network generates a further dilution of hydrogen, generating areas with lower hydrogen share as the distance from the injection point increases. In particular, with respect to the previous simulation, the total share of users who are reached by hydrogen concentration at least higher than $1 \%$ are $44 \%$ of the total. However, only $16 \%$ are reached by an exact $10 \%$ hydrogen blend. The remaining $11 \%$ and $17 \%$ are reached by hydrogen-natural gas mixtures with hydrogen share between $10 \%$ and $5 \%$ and between $5 \%$ and $1 \%$ respectively. 


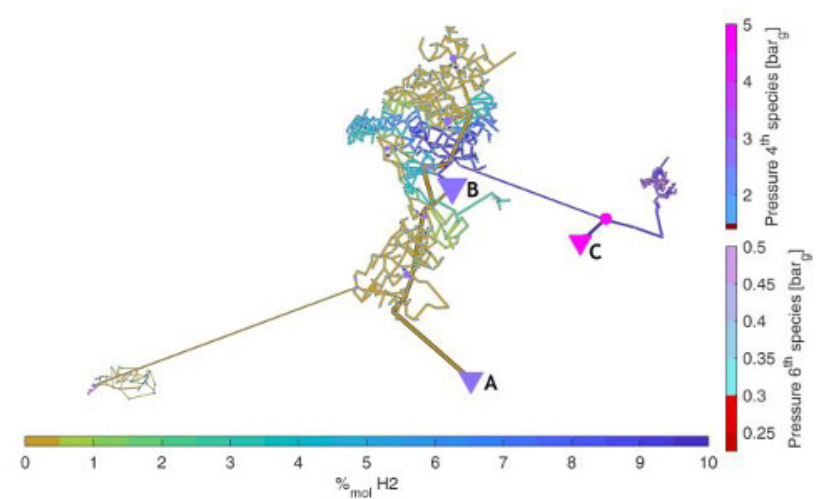

Fig. 3. Fluid-dynamic and quality tracking results of the second simulated scenario: the pressure regulation station in which hydrogen blending is performed has a higher pressure set-point.

\section{Conclusion}

The hydrogen injection within the gas network at the distribution level has the advantage of delimiting the impacts on gas quality variations to a local level, without affecting the higher pressure transmission infrastructure. This aspect can ease the roll-out of blending projects without the need for national or international standardization process and technical regulation revision. However, the magnitude of acceptable hydrogen injection flow rates, which is already lower, may be further lowered because of the peculiarity of the fluid-dynamic equilibrium of the distribution network, especially in those networks with multiple gas entry points.

In this work, these aspects have been highlighted and an approach in order to alleviate these bottlenecks has been proposed. The coordinated management of pressure set-points of the gas reduction stations at all pressure tiers is shown to be a valid management scheme in order to enhance the hydrogen injection and distribution throughout the network. The specific example showed that the hydrogen injection rate has been increased by a factor of 2.5 by setting different pressure set-points, with negligible impacts on grid operations.

The multi-component fluid-dynamic modeling showed also the increase of complexity the distribution grids are going to face in the framework of hydrogen injection, in terms of gas quality distribution. The gas network operator will have to handle networks with nonhomogeneous quality as well as dynamically manage the possible different sources of gases, by tuning pressures and gas flows. The evolution towards a smarter gas network will be fundamental for the management of the multi-gas networks of the future.

\section{References}

[1] ewi Energy Research \& Scenarios gGmbH, The energy market in 2030 and 2050 - The contribution of gas and heat infrastructure to efficient carbon emission reductions (2018).

[2] Pöyry, Fully decarbonising Europe's energy system by 2050 Decarbonising Europe's energy system (2018).

[3] I. Staffell, D. Scamman, A. Velazquez Abad, P. Balcombe, P.E. Dodds, P. Ekins, N. Shah, K.R. Ward, Energy Environ. Sci. 12 (2019)

[4] Enagás, Energinet, Fluxys Belgium, Gasunie, GRTgaz, NET4GAS, OGE, ONTRAS, Snam, Swedegas, Teréga, European Hydrogen Backbone (2020)

[5] UNI/EN, UNI 16726:2018, (2018).

[6] K. Altfeld, D. Pinchbeck, Gas Energy (2013)

[7] Marcogaz, Overview of available test results and regulatory limits for hydrogen admission into existing natural gas infrastructure and end use (2019).

[8] E. Giacomazzi, G. Messina, Energia, Ambient. e Innov.(2021)

[9] EIGA, Hydrogen transportation pipelines (2004)

[10] A. Huszal, J. Jaworski, Energies (2020)

[11] Marcogaz, Odorisation Of Natural Gas And Hydrogen Mixtures (2021).

[12] J. Jaworski, P. Kułaga, T. Blacharski (2020)

[13] GRTgaz, Report on the impact of renewable gases, and mixtures with natural gas, on the accuracy, cost and lifetime of gas meters. Literature overview for renewable gases flowmetering. (2020).

[14] M.J. Kippers, J.C. De Laat, R.J.M. Hermkens, J.J. Overdiep, A. Van Der Molen, W.C. Van Erp, A. Van Der Meer, Int. Gas Res. Conf. 2011 Proc., Seoul (2011)

[15] T. Isaac, Clean Energy (2019)

[16] M. Abeysekera, J. Wu, N. Jenkins, M. Rees, Appl. Energy. 164 (2015)

[17] M. Cavana, A. Mazza, G. Chicco, P. Leone, Appl. Energy. 290 (2021)

[18] C. Fiebig Hielscher A. Span R. Gulin A. Rickelt S. Schley P., Int. Gas Union Res. Conf. (2014)

[19] P. Schley, A. Hielscher, C.F.M. Sc, S. Rickelt, World Gas Conference, (2018)

[20] M. Chaudry, N. Jenkins, G. Strbac, Electr. Power Syst. Res. 78 (2008)

[21] A.J. Osiadacz, M. Chaczykowski, Chem. Eng. J. 81 (2001)

[22] S. V. Patankar, D.B. Spalding, Int. J. Heat Mass Transf. 15 (1972)

[23] M. Cavana, Gas network modelling for a multigas system, Politecnico di Torino, 2020. 